MATEC Web of Conferences 3, 01085 (2013)

DOI: $10.1051 /$ matecconf/20130301085

(C) Owned by the authors, published by EDP Sciences, 2013

\title{
Rimonabant dimorphism, much ado about almost nothing
}

\author{
M.-A. Perrin ${ }^{1}$, M. Bauer ${ }^{1}$, M. Barrio ${ }^{2}$, J.-Ll. Tamarit ${ }^{2}$, R. Céolin ${ }^{2,3}$, and I.B. Rietveld ${ }^{3}$ \\ ${ }^{1}$ Sanofi, France \\ ${ }^{2}$ Grup de Caracterització de Materials (GCM), Universitat Politècnica de Catalunya, Barcelona, Spain \\ ${ }^{3}$ EAD Physico-chimie Industrielle du Médicament (EA 4066), Université Paris Descartes, Paris, France
}

\section{Introduction}

Molecular polymorphism is being studied for almost 2 centuries, if the discovery of two solid forms of benzamide in 1832 by Justus Liebig and Friedrich Wöhler is taken as the starting point. Even though considerable progress has been made since then - the description of classical thermodynamics by Gibbs, its application by Bakhuis-Roozeboom, and much more recently the growing capability to calculate and thus predict energy differences between crystal structures, to mention a few - in many cases, one is still taken by surprise, when a polymorphic form is discovered. Moreover, one does not escape a thorough experimental analysis of the polymorphic forms to determine with certainty which of them is the more stable form under the required conditions.

Rimonabant,

5-(4-chlorophenyl)-1-(2,4dichlorophenyl)-4-methyl- $N$-1-piperidinyl-1 $H$-pyrazole3-carboxamide) presents such a case study. It used to be an active pharmaceutical ingredient (API), which has been taken from the market due to its side effects. During the development of the drug, a second solid form was discovered and it had been very difficult to establish which of the solid forms is the more stable one. This was mainly due to the small difference in Gibbs energy that exists between the two forms.

\section{Experimental}

Measurements were carried out with a TA Instruments Q1000, a TA Instruments Q100, and a PerkinElmer Pyris Diamond analyzer. They were calibrated with indium $\left(\mathrm{T}_{\text {fus, onset }}=429.8 \mathrm{~K}, \Delta_{\text {fus }} \mathrm{H}=28.71 \mathrm{~J} \mathrm{~g} \mathrm{~g}^{-1}\right)$ and lead $\left(\mathrm{T}_{\text {fus, }}\right.$ ${ }_{\text {onset }}=600.7 \mathrm{~K}$ ). Samples between 1.5 and $5 \mathrm{mg}$ were placed in sealed aluminum pans. The heating rate was 5 $\mathrm{K} \min ^{-1}$.

X-ray single crystal diffraction (XRSCD) data were recorded on a Bruker Smart Apex single crystal diffractometer. For temperature dependent measurements, an Oxford Cryosystems nitrogen cryostat (Cryostream Plus) was added to the equipment described above. The cryostat allows XRSCD experiments to be carried out in a temperature range from 200 to $400 \mathrm{~K}$.

High-Pressure Differential Thermal Analysis (HPDTA) measurements were carried out with a heating rate of $2 \mathrm{~K} \mathrm{~min}^{-1}$ using an in-house built apparatus similar to that designed by Würflinger [1] with operating ranges between $298-473 \mathrm{~K}$ and $0-300 \mathrm{MPa}$. To ensure that inpan volumes were free from residual air, specimens were mixed with an inert perfluorinated liquid (Galden ${ }^{\circledR}$, from Bioblock Scientifics, Illkirch, France) as a pressuretransmitting medium, and the mixtures were sealed into cylindrical tin pans, typically containing about $40 \mathrm{mg}$ of sample.

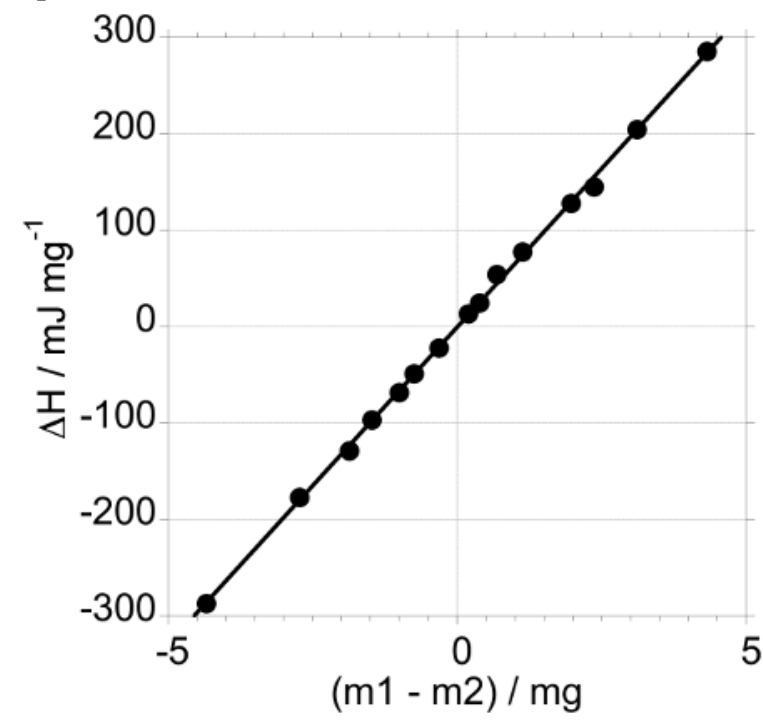

Figure 1. Simultaneous melting of form I (at the sample position) and form II (at the reference position)[2]. The total enthalpy (i.e. the difference in melting enthalpies, $\Delta_{\text {fus }} \mathrm{H}(\mathrm{I})-$

$\left.\Delta_{\text {fus }} \mathrm{H}(\mathrm{II})\right)$ of the event has been plotted against the difference in mass between the two capsules, $\mathrm{r}^{2}=0.9996$.

\section{Results}

The melting point of form I, $T_{\text {fus, },}$, is $428.2 \pm 0.3 \mathrm{~K}$ with an enthalpy change, $\Delta_{\text {fus }} h_{\mathrm{I}}$, of $67.4 \pm 2.1 \mathrm{~J} \mathrm{~g}^{-1}$. Form II has a melting point, $T_{\text {fus,II }}$, of $429.0 \pm 0.5 \mathrm{~K}$ and an enthalpy change, $\Delta_{\text {fus }} h_{\mathrm{II}}$, of $68.4 \pm 2.6 \mathrm{~J} \mathrm{~g}^{-1}$. The melting point and the enthalpy change are the same within error for both 
forms. However the sign of the difference in melting temperature and melting enthalpy could be determined unequivocally, as explained below.

Form I was placed in the sample position of the DSC and form II in the reference position. For each measurement the initial slope of the combined melting peak went up (endotherm up), indicating that form I melts at a slightly lower temperature than form II. Tracing the observed enthalpies as a function of difference in mass $\left(\mathrm{m}_{\mathrm{I}}-\mathrm{m}_{\mathrm{II}}\right)$ leads to the following equation (Figure 1$)$ :

$$
\Delta\left(\Delta_{\text {fus }} \mathrm{H}\right)\left(\mathrm{mJ} \mathrm{mg}^{-1}\right)=65.9(5)[\mathrm{m} 1-\mathrm{m} 2]-0.60(1.17)
$$

It can be seen that the enthalpy difference between the two solid phases at the melting temperature is $-0.60 \mathrm{~J} \mathrm{~g}^{-1}$, with form I having the lowest enthalpy. In addition, comparison of individual fusion enthalpy measurements of the two solid forms consistently demonstrated that form II has a slightly larger melting enthalpy. Thus, it could be established unequivocally that $\mathrm{T}_{\text {fus,I }}-\mathrm{T}_{\text {fus,II }}$ and $\Delta_{\text {fus }} \mathrm{H}_{\mathrm{I}}-\Delta_{\text {fus }} \mathrm{H}_{\mathrm{II}}$ are negative, even though there is a large relative uncertainty over their numerical values.

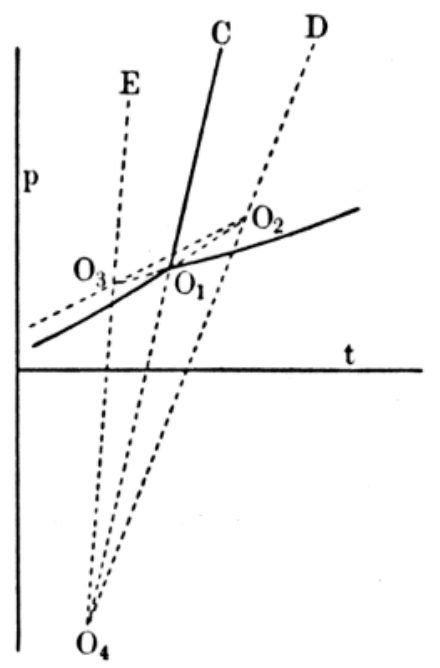

Figure 2. Schematic pressure-temperature phase diagram of a monotropic system by Bakhuis-Roozeboom [4]. Solid lines are the stable phase transitions. 'C' stable fusion of form II, ' $\mathrm{E}$ ' metastable fusion of form I, ' $\mathrm{D}$ ' metastable solid-solid transition. ' $\mathrm{O}$ ' triple points: $\mathrm{O}_{1}$ form II - liquid - vapor triple point.

The slopes of the solid-liquid equilibria have been determined by HP-DTA resulting in $2.5 \mathrm{MPa} \mathrm{K}^{-1}$ for $\mathrm{I} \rightarrow$ $\mathrm{L}$ and $2.4 \mathrm{MPa} \mathrm{K}^{-1}$ for II $\rightarrow \mathrm{L}$. Thus, the two equilibria are diverging and the rimonabant system is overall monotropic. This has been confirmed by the topological approach [3] using DSC data, specific volumes from the $\mathrm{X}$-ray diffraction measurements and the Clapeyron equation. A schematic pressure-temperature phase diagram (originally drawn by Bakhuis-Roozeboom [4]) can be found in Figure 2.

\section{Conclusion}

The differences of the physical properties of the dimorphic forms of rimonabant are very small. This has complicated the investigation to determine the more stable form under ordinary conditions. The combination of direct measurement by high-pressure differential thermal analysis and the topological approach eventually led to an unambiguous interpretation of the phase behavior. Rimonabant is overall monotropic with form II as the only stable phase.

\section{References}

1. A. Würflinger, Ber. Bunsen Phys. Chem. 791195 (1975)

2. S. Toscani, S. Thoren, V. Agafonov, R. Céolin, J. Dugué, Pharm. Res. 121453 (1995)

3. R. Céolin, I. B. Rietveld, J. Therm. Anal. Calorim. 102357 (2010)

4. H. W. Bakhuis Roozeboom, Die heterogenen Gleichgewichte vom Standpunkte der Phasenlehre. Erstes Heft: Die Phasenlehre - Systeme aus einer Komponente, Friedrich Vieweg und Sohn, Braunschweig, 1901 